\title{
Combined effect of end-rounded versus tapered bristles and a dentifrice on plaque removal and gingival abrasion
}

Leonardo Stephan CAPOROSSI (a) Danilo Antonio Milbradt DUTRA ${ }^{(a)}$ Maritieli Righi MARTINS(a) Emilia Pithan PROCHNOW(a) Carlos Heitor Cunha MOREIRA(b) Karla Zanini KANTORSKI(b)

(a) Universidade Federal de Santa Maria - UFSM, School of Dentistry, Department of Periodontology, Santa Maria, RS, Brazil.

(b) Universidade Federal de Santa Maria - UFSM, School of Dentistry, Department of Stomatology, Santa Maria, RS, Brazil.

Declaration of Interests: The authors certify that they have no commercial or associative interest that represents a conflict of interest in connection with the manuscript.

Corresponding Author:

Karla Zanini Kantorski

E-mail: kzkantorski@hotmail.com

Submitted: May 30, 2015

Accept for publication: Oct 14, 2015

Last revision: Nov 19, 2015
Abstract: Two previous clinical studies evaluated the effect of end-rounded versus tapered bristles of soft manual brushes on the removal of plaque and gingival abrasion. However, the combined effect of an abrasive dentifrice on these outcomes has yet to be understood. The purpose of the present study was to compare the incidence of gingival abrasion and the degree of plaque removal obtained after the use of toothbrushes with tapered or end-rounded bristles in the presence or absence of an abrasive dentifrice. The study involved a randomized, single-blind, crossover model $(n=39)$ with a split-mouth design. Subjects were instructed to refrain from performing oral hygiene procedures for 72 hours. Quadrants were randomized and subjects brushed with both types of toothbrushes using a dentifrice (relative dentin abrasion $= \pm 160$ ). Plaque and gingival abrasion were assessed before and after brushing. After 7 days, the experiment was repeated without the dentifrice. The average reduction in plaque scores and the average increase in the number of abrasion sites were assessed by repeated-measures ANOVA and Bonferroni's post-hoc tests. End-rounded bristles removed significantly more plaque than tapered bristles, regardless of the use of a dentifrice. The dentifrice did not improve plaque removal. In the marginal area (cervical free gingiva), no difference in the incidence of gingival abrasion was detected between toothbrush types when used with a dentifrice $(p \geq 0.05)$. However, the dentifrice increased the incidence of abrasion $(p<0.001)$, irrespective of the toothbrush type tested. End-rounded bristles therefore removed plaque more effectively without causing a higher incidence of gingival abrasion when compared with tapered bristles. An abrasive dentifrice can increase the incidence of abrasion, and should be used with caution by individuals who are at risk of developing gingival recession.

Keywords: Periodontal Diseases; Gingival Recession; Oral Hygiene; Dental Plaque.

\section{Introduction}

The occurrence of gingival recession among subjects with a good standard of oral hygiene ${ }^{1,2}$ suggests that it may be the result of traumatic toothbrushing. The aetiology of gingival recession is multifactorial. It involves anatomical and iatrogenic factors, in addition to pathologic conditions associated with dental plaque, such as periodontitis. ${ }^{3}$ The 
possibility that toothbrushing trauma may be a contributing factor to gingival recession has been considered, although the scientific evidence to confirm this hypothesis appears to be inconsistent. ${ }^{4,5,6}$ In a recent systematic review, Heasman et al. ${ }^{6}$ showed that the most frequent toothbrushing factors associated with gingival recession were frequency of oral hygiene procedures, use of a horizontal or scrubbing method, bristle hardness, toothbrushing duration, and frequency of toothbrush replacement.

Soft tissue lesions caused by oral hygiene procedures are denominated gingival abrasion, and are usually not detected during clinical examination. For research purposes, a disclosing solution is used to identify gingival lesions of epithelial desquamation. ${ }^{78}$

The literature has shown that brushes with medium and stiff bristles remove more plaque than soft brushes,

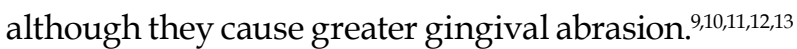
In addition, medium-hard toothbrushes were found to be a significant risk factor for the incidence of gingival fissures. ${ }^{14}$ With regard to the type of bristle finish, there is evidence that straight bristles cause more damage to hard and soft tissues when compared to the end-rounded variety. ${ }^{13,15,16}$ Soft brushes with tapered or conical filaments were recently introduced. The diameter of the body of these filaments is similar to that of the filaments of a conventional soft brush, but the diameter of the filament tip is significantly smaller. In principle, this could result in effective plaque removal from the gingival sulcus and proximal surfaces without damaging the gingiva.

Two previous studies have evaluated soft brushes with tapered filaments. ${ }^{8,17}$ In the first study, where brushing was performed only with water, Dorfer et al. ${ }^{17}$ found that the toothbrushes with tapered filaments showed significantly higher plaque-removing efficacy compared to those with filaments with a standard flat trim. However, the authors doubted the clinical relevance of this difference. In the second study, Versteeg et al. ${ }^{8}$ evaluated toothbrushes with tapered filaments and end-rounded cylindrical filaments in regard to plaque removal, gingival bleeding and gingival abrasion using an experimental gingivitis model and a dentifrice with low abrasiveness (relative dentin abrasion, $\mathrm{RDA}= \pm 76$ ). The authors observed that tapered filaments were less effective in removing plaque and reducing bleeding. No difference was observed between toothbrush types in regard to gingival abrasion.

To our knowledge, no study has been conducted on the behavior of soft toothbrushes with tapered versus rounded filaments associated with a dentifrice of higher abrasiveness, in terms of gingival abrasion. Understanding the effect of a dentifrice is important because its abrasiveness may aggravate gingival abrasion, even when used with a soft brush.

Therefore, the objective of this study was to evaluate the effect of soft toothbrushes with tapered bristles versus that of soft toothbrushes with end-rounded bristles on the incidence of gingival abrasion and on the degree of plaque removal obtained in the presence or absence of an abrasive dentifrice. Two hypotheses were considered: 1 , self-performed plaque control with a toothbrush with end-rounded bristles would result in greater gingival abrasion compared to one with tapered bristles; 2 , the use of abrasive toothpaste would aggravate gingival abrasion.

\section{Methodology}

\section{Experimental design}

This study was a split-mouth, crossover (two periods), single-blind, randomized clinical trial. In the first period, the subjects brushed their teeth with a dentifrice. In the second period, the same subjects brushed their teeth only with water. Each subject used the two toothbrush types during each period in randomized quadrants.

The study had three-phases: a familiarization phase (subjects became acquainted with the use of both toothbrushes), an abstention phase (no oral hygiene for 72 hours), and an assessment phase (assessment of gingival abrasion and plaque removal).

\section{Study population}

The potential participants were non-dental students, with at least 18 years of age, registered at the Universidade Federal de Santa Maria - UFSM in Santa Maria, RS, Brazil. All subjects were recruited from July to November, 2012. 
Subjects were included if they had a papilla completely filling the interdental space, at least 24 teeth, and a maximum of $15 \%$ of sites with gingival bleeding after probing. Subjects were excluded if they had orthodontic appliances or retention; fixed prostheses; were smokers, diabetics, pregnant or lactating; or if they had periodontitis. Periodontitis was defined as a loss $\geq 3 \mathrm{~mm}$ of interproximal attachment in two or more non-adjacent teeth ${ }^{18}$ with probing depths $\geq 3 \mathrm{~mm}$ associated with bleeding on probing in at least one site, with the exception of the distal aspect of the terminal teeth in each arch.

Subjects were informed about the purpose of the study in their classrooms. Volunteers were questioned regarding their overall health and habits, and they were screened to verify their eligibility.

\section{Sample size}

Gingival abrasion was considered the primary outcome for calculating the sample size. There are no studies in the literature that evaluate the effect of soft brushes with tapered versus end-rounded bristles using an abrasive dentifrice. The study's sample size was therefore determined based on pilot studies. The parameters used for determining the sample size were: a difference of 6 in the number of sites with gingival abrasion after brushing, with a standard deviation of $10(\Delta \pm S D)$, a power of $80 \%$, a significance level of $5 \%$, and an expected dropout rate of $15 \%$; therefore, 39 patients were required.

\section{Ethical considerations}

All of the subjects signed an informed consent form. The study was performed in accordance with the Declaration of Helsinki and was approved by the Research Ethics Committee of the Universidade Federal de Santa Maria - UFSM, RS, Brazil (approval no. 04322912.6.0000.5346).

\section{Training and calibration}

The examinations were performed by a single examiner (LC), who was calibrated to assess gingival abrasion ${ }^{19}$ and stained plaque ${ }^{20}$ (Turesky's modification of the Quigley-Hein Plaque Index; ${ }^{20}$ Turesky et al. ${ }^{21}$ ). The evaluation of plaque and gingival abrasion included the use of a disclosing solution. Due to the clinical nature of the index used, the calibration was performed using photography. However, for the period of study, the evaluations of gingival abrasion and stained plaque were performed during the clinical examination.

Seven subjects, not included in the study, were instructed not to perform oral hygiene procedures for a period of 72 hours. After this waiting period, the plaque and mucosa were stained and photographed using a digital camera (Camera: Canon EOS Digital Rebel XT, Lens: Canon 100 mm f/2.8 Macro Lens, and Flash: Canon MR-14EX Ring Lite, Canon, Newport News, USA) using cheek retractors. After the subjects brushed their teeth using soft toothbrushes and fluoridated toothpaste for two minutes, the plaque and mucosa were stained again. New photographs were taken.

Calibration was performed on the photographs on two occasions, with an interval of 1 hour between assessments. Kappa coefficients were 0.94 and 0.73 for plaque index and gingival abrasion, respectively.

\section{Assessment methods}

Dental plaque was clinically assessed at six sites per tooth. The teeth were air-dried and isolated with cotton rolls. Plaque was stained with a 2-tone disclosing solution (Young Dental, Earth City, USA) and was recorded on a scale of 0 to $5(0=$ no plaque, 5 = plaque covering more than two-thirds of the tooth surface).${ }^{21}$ Each quadrant was stained using a new cotton swab moistened with the dye.

Gingival abrasion was evaluated using the same 2-tone disclosing solution to visualize the abraded areas of the oral epithelium. Each quadrant was stained using a new cotton swab moistened with the dye. The gingival tissue was divided into two areas: marginal (cervical free gingiva), and interdental (papillary free gingiva). ${ }^{8}$ The examiner (LC) clinically determined the number of lesions and their location. The buccal and lingual gingiva was evaluated, except for the regions around third molars and central incisors. The rationale for not including the region around central incisors was to avoid an overlapping of adjacent quadrants during brushing. 


\section{Experimental period}

Before beginning the experiment, the participants received a prophylaxis and coronary polishing. The experimental period consisted of 3 phases: familiarization phase, abstention phase, and assessment phase.

\section{Familiarization phase}

The objective of this phase was to familiarize the participants with the two brush types used in the experiment. The familiarization period was 10 days. ${ }^{7}$ Each subject received two soft toothbrushes: the first with end-rounded filaments (Oral- $\mathrm{B}^{\circledR}$ Indicator ${ }^{\circledR}$ Plus, size 35, Gross-Gerau, Germany), whose handle was covered by blue tape, and the second with tapered filaments (Colgate $360^{\circ}$ Deep Clean, São Bernardo do Campo, Brazil), whose handle was covered by red tape. The brush characteristics are presented in Table 1 and Figure 1.

For 10 days, the subjects brushed their teeth by alternating between the toothbrush types every other day. No specific technique of tooth brushing was suggested. A colorful calendar printed with the respective colors of the toothbrushes (blue and red) was provided. Thus, each calendar day was marked with a color indicating the particular toothbrush to be used.

\section{Abstention phase}

The subjects were instructed to refrain from any oral hygiene procedures (mechanical or chemical) for 72 hours. Subsequently, the presence of stained plaque and gingival abrasion was assessed by the investigator (LC; pre-brushing examination).

\section{Assessment phase}

After the pre-brushing examination, the examiner (LC) left the office to ensure the blinding of the experimental groups. A researcher (MRM) used a coin flip to randomize the contralateral quadrants (first and third, second and fourth) of the mouth to be brushed with end-rounded or tapered toothbrush filaments, using a stannous fluoride-containing dentifrice (RDA $= \pm 160$; Oral- $B^{\circledast}$ Pro-Saúde, Procter \& Gamble, Gross-Gerau, Germany).

The researcher (MRM) applied the toothpaste starting from a fixed point and then covering the entire width of the bristle area, in order to standardize the amount used $( \pm 0.5 \mathrm{~g})$. Participants brushed their teeth without using a mirror, which prevented them from visualizing the stained areas. ${ }^{22}$ Brushing was performed for $30 \mathrm{~s}$ in each quadrant without providing specific instructions on technique: $15 \mathrm{~s}$ for the buccal and $15 \mathrm{~s}$ for the lingual surfaces, ${ }^{7,22}$ The researcher monitored the brushing time using a stopwatch. Next, the examiner (LC) assessed the presence of gingival abrasion and stained plaque (post-brushing examination).

After a 7 day period (washout), the second part of the study was conducted. The same procedures as described in the first period were performed; however, brushing was performed only with water.

Four experimental groups were evaluated: soft brush with end-rounded filaments and toothpaste, soft brush with end-rounded filaments and water, soft brush with tapered filaments and toothpaste, and soft brush with tapered filaments and water.

\section{Outcomes}

The difference between groups in terms of mean number of sites with gingival abrasion was the primary

Table 1. Toothbrush characteristics.

\begin{tabular}{lcc}
\hline Toothbrush feature & Tapered filaments & End-rounded filaments \\
Head length & $2.88 \mathrm{~cm}$ & $2.65 \mathrm{~cm}$ \\
Head width & $1.31 \mathrm{~cm}$ & $1.17 \mathrm{~cm}$ \\
Number of tufts & 38 & 30 \\
Number of filaments & 38 & 40 \\
Filament base diameter & $\pm 0.11 \mathrm{~mm}$ & $\pm 0.12 \mathrm{~mm}$ \\
Filament tip diameter & $\pm 0.02 \mathrm{~mm}$ & $\pm 0.12 \mathrm{~mm}$
\end{tabular}



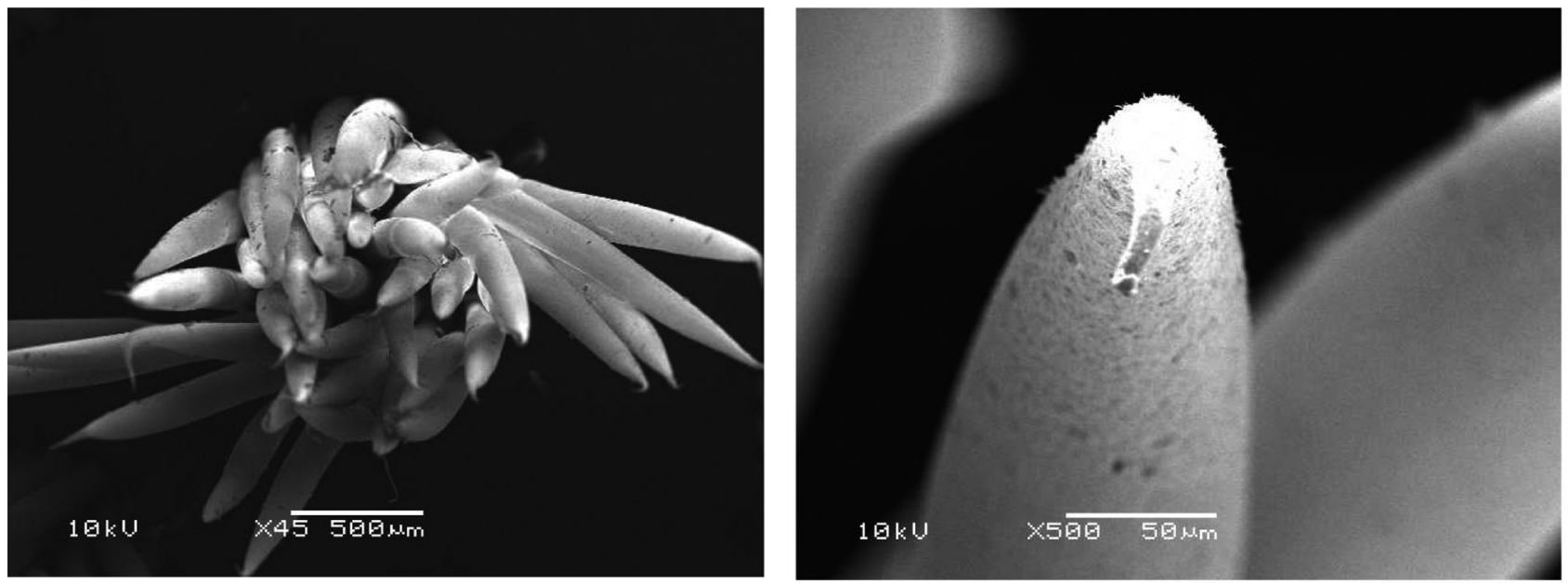

Scanning electron microscopic image of the tapered filaments ( $\times 45$ and $x 500$ )
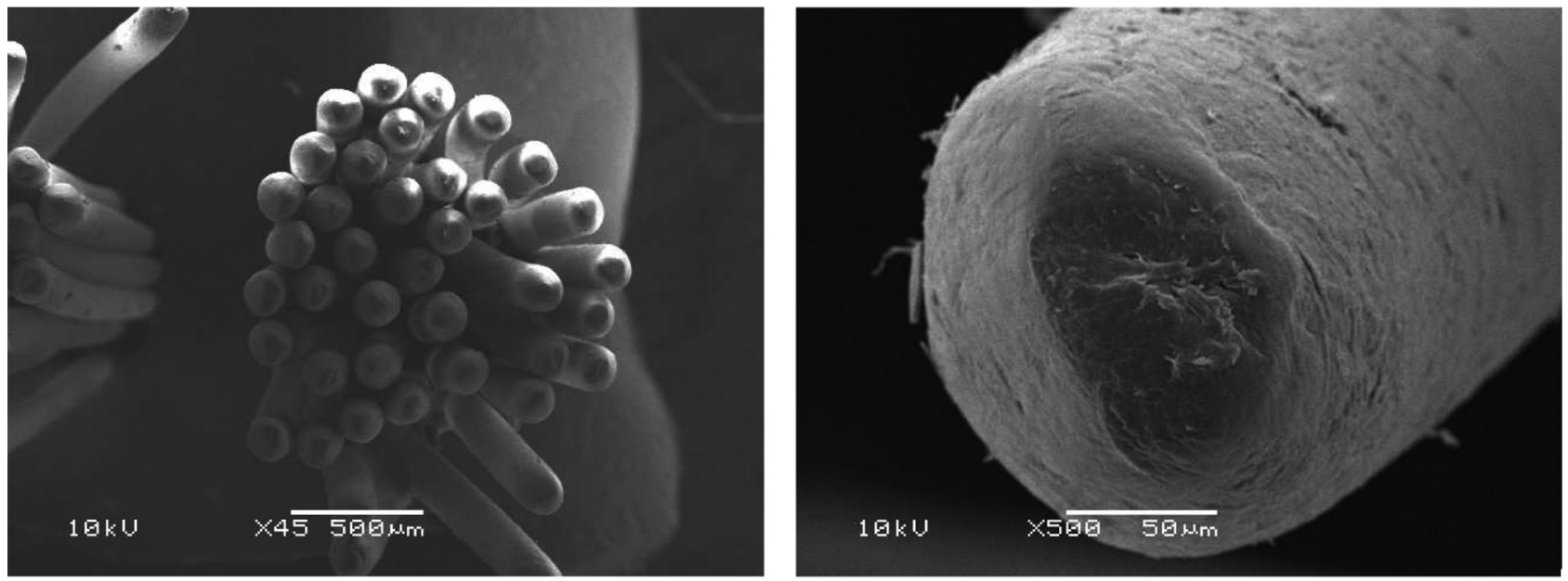

Scanning electron microscopic image of the end-rounded filaments ( $x 45$ and $x 500)$.

Figure 1. Toothbrush characteristics.

outcome. The mean reduction in plaque scores was the secondary outcome.

\section{Data analysis}

Each subject was a unit of analysis. The average percentage of sites with gingival abrasion and the mean plaque scores were calculated before and after brushing to determine the incidence of abrasion and the reduction in the average plaque scores after brushing ( $\Delta \pm$ standard deviation). Pre-toothbrushing data were analyzed using one-way ANOVA and the post-hoc Tukey test. Abrasion incidence values were calculated for the marginal and interdental areas. Plaque reduction values were calculated for free and interproximal surfaces. Differences between groups were analyzed using repeated-measures ANOVA and the post-hoc Bonferroni test. Tooth type (anterior, premolar, and molar) and site (buccal versus lingual) were used for explorative analysis. Differences between tooth types and sites were analyzed using the Kruskal-Wallis and Mann-Whitney tests, respectively. The statistical analyses were performed using SPSS, v.13.0 software (SPSS, Inc., Chicago, USA). Differences were considered statistically significant at $p<0.05$.

\section{Results}

A total of 53 subjects were evaluated from July to November, 2012. Of these, 12 patients were not eligible 
(11 due to orthodontic appliances, 1 due to a fixed prosthesis) and 2 chose not to participate. Thirty-nine patients participated in the study (Figure 2). The experiment was completed in December of 2012.

The mean age of the subjects was $23.5 \pm 2.66$ years old, with 21 female and 18 male participants. Table 2 presents the pre-toothbrushing data for plaque index and mean percentage of sites with gingival abrasion. No statistically significant differences were observed between experimental groups at the pre-brushing examination ( $p>0.05$ for all cases).

The data for average plaque reduction $(\Delta \pm$ standard deviation) and $95 \%$ confidence interval $(95 \% \mathrm{CI})$ for the experimental groups according to tooth surface location are shown in Table 3. The brushes with end-rounded bristles removed more plaque from tooth surfaces (including the proximal surfaces) than those with tapered bristles, irrespective of whether or not toothpaste was used. The use of a dentifrice did not significantly increase plaque removal, regardless of the type of toothbrush used.

Table 4 presents the mean percentage increase $(\Delta \pm \mathrm{SD})$ and $95 \% \mathrm{CI}$ for the number of sites with gingival abrasion according to lesion location. A higher incidence of abrasion was observed in the group that used toothbrushes with end-rounded bristles and toothpaste, regardless of lesion location. However, a statistically significant difference was observed only between end-rounded bristles plus toothpaste and tapered bristles plus water $(p<0.05)$. In the marginal area, the use of dentifrice significantly increased the incidence of abrasion, regardless of the type of toothbrush used. When brushing was performed with water, the brushes with end-rounded bristles caused significantly greater abrasion compared to those with tapered bristles. When the dentifrice was used, no difference was observed between toothbrush types. In the papilla, a statistically significant difference was observed only between end-rounded bristles plus toothpaste and tapered bristles plus water.

Figure 3 presents the mean percentage increase $(\Delta \pm \mathrm{SD})$ in the number of sites with gingival abrasion according to tooth type and site. Premolars showed a significantly higher incidence of abrasion than anterior teeth in the end-rounded filaments

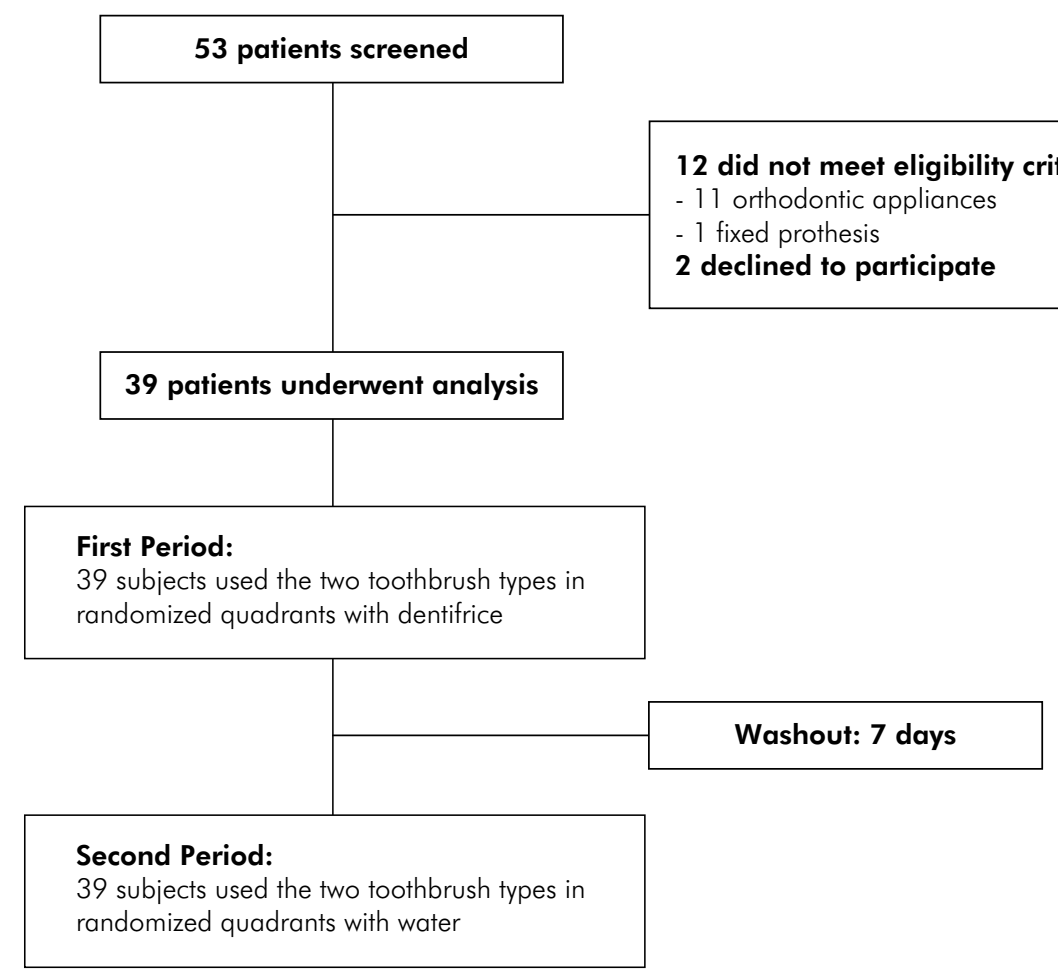

Figure 2. Flowchart of the study design. 
Table 2. Pre-toothbrushing data. Quigley-Hein mean plaque index (SD) and mean percentage of sites with gingival abrasion (SD).

\begin{tabular}{lccc}
\hline & Experimental Groups & Quigley-Hein mean plaque index & Mean percentage of sites with gingival abrasion \\
\hline Dentifrice & End-rounded filament & $3.04(0.45)$ & $7.06(2.64)$ \\
& Tapered filament & $3.06(0.45)$ & $7.35(2.70)$ \\
Water & End-rounded filament & $3.09(0.56)$ & $6.95(2.62)$ \\
& Tapered filament & $3.11(0.57)$ & $7.02(2.82)$ \\
\hline
\end{tabular}

Columns: one-way ANOVA and post-hoc Tukey tests. No statistically significant differences were observed at the pre-brushing examination ( $p>0.05$ for all cases).

Table 3. Mean reduction of plaque (SD) and $95 \% \mathrm{Cl}$ according to the tooth surface location.

\begin{tabular}{|c|c|c|c|c|}
\hline \multirow{2}{*}{ Location } & \multicolumn{2}{|c|}{ Tapered filament } & \multicolumn{2}{|c|}{ End-rounded filament } \\
\hline & Water & Dentifrice & Water & Dentifrice \\
\hline Overall & 1.59 (0.45)\# 1.44-1.75 & $1.63(0.50) \# 1.46-1.80$ & $1.84(0.54)^{*} 1.66-2.02$ & $1.92(0.51)^{*} 1.75-2.10$ \\
\hline Free surface & $1.79(0.52) \# 1.61-1.96$ & $1.82(0.52) \#^{*} 1.65-2.00$ & $2.00(0.58)^{*} \& 1.80-2.02$ & $2.03(0.50) \& 1.85-2.22$ \\
\hline Proximal surface & 1.49 (0.47)\# 1.33-1.64 & 1.54 (0.53)\# 1.36-1.72 & $1.77(0.54)^{*} 1.58-1.95$ & $1.88(0.52)^{*} 1.70-2.05$ \\
\hline
\end{tabular}

Line: repeated measures ANOVA and post-hoc Bonferroni tests.

Same symbols: no statistically significant difference $(p \geq 0.05)$

Different symbols: statistically significant difference $(p<0.05)$

Table 4. Mean percentage increase (SD) and $95 \% \mathrm{Cl}$ in the number of sites with gingival abrasion according to lesion location.

\begin{tabular}{lcccc}
\hline \multirow{2}{*}{ Location } & \multicolumn{2}{c}{ Tapered filament } & \multicolumn{2}{c}{ End-rounded filament } \\
\cline { 2 - 5 } & Water & Dentifrice & Water & Dentifrice \\
\hline Overall & $6.7(8.2) \# 0.4-0.95$ & $9.8(13.1) \#^{*} 5.5-14.3$ & $10.2(9.7) \# * 7.0-13.5$ & $12.6(11.3)^{*} 8.9-16.4$ \\
Marginal & $6.6(12.3) \# 2.5-10.7$ & $17.1(15.4)^{*} \& 12.0-22.3$ & $13.9(10.9)^{*} 10.3-17.6$ & $22.0(12.8) \& 17.4-26.7$ \\
Interdental & $6.7(8.2) \# 0.4-9.5$ & $9.8(13.1) \#^{*} 5.5-14.3$ & $10.2(9.7) \#^{*} 0.7-13.5$ & $12.6(11.3)^{*} 8.9-16.4$ \\
\hline
\end{tabular}

Line: repeated measures ANOVA and post-hoc Bonferroni tests.

Same symbols: no statistically significant difference $(p \geq 0.05)$

Different symbols: statistically significant difference $(p<0.05)$

Marginal: cervical free gingiva.

Interdental: papillary free gingiva.

plus dentifrice $(p=0.027)$, end-rounded filaments plus water $(\mathrm{p}=0.046)$, and tapered filaments plus dentifrice $(p=0.042)$ groups. Additionally, the buccal sites of premolars presented a significantly higher incidence of abrasion than lingual sites when dentifrice was used, irrespective of toothbrush type ( $p=0.034$ for end-rounded filaments; $p=0.033$ for tapered filaments).

\section{Discussion}

The results of this study show that a soft brush with end-rounded bristles has advantages over one with tapered bristles for maintaining oral hygiene. When used along with a dentifrice, the end-rounded bristles removed more plaque from tooth surfaces (including proximal surfaces), and did not increase the incidence of gingival abrasion when compared to tapered bristles. These results fail to confirm the initial hypothesis that suggested end-rounded bristles would cause greater gingival abrasion. The other hypothesis, that the use of a dentifrice would increase gingival abrasion, was not confirmed when abrasion was evaluated irrespective of site. However, when considering the incidence of abrasion with respect to the marginal area, the use of a dentifrice resulted in a significant 


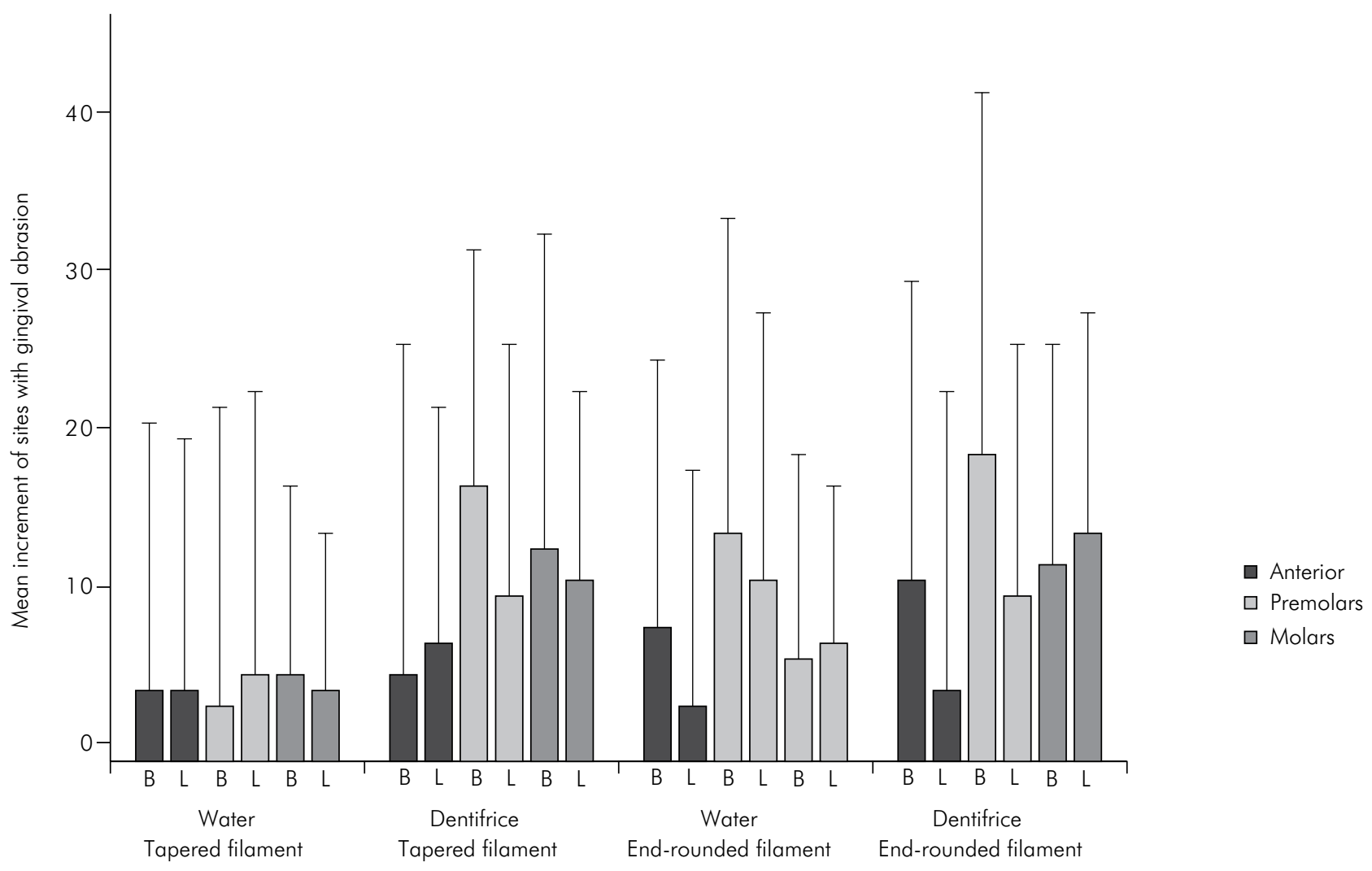

Figure 3. Mean percentage increase $(\Delta \pm \mathrm{SD})$ in the number of sites with gingival abrasion according to tooth type and site (B: buccal site, L: lingual site).

increase in abrasive lesions, regardless of the type of toothbrush used.

The use of fluoride toothpaste is essential for preventing caries. During brushing, rubbing of the dentifrice on the mucosa can result in the removal of salivary glycoproteins, thus causing epithelial cell death. ${ }^{23}$ Moreover, the abrasive dentifrice components (silica, alumina, calcium carbonate), which are related to a dentifrice's degree of abrasiveness, can cause mechanical abrasion of the epithelium. According to Verteeg et al., ${ }^{24}$ brushing with a dentifrice did not result in increased gingival abrasion. Our results partially agree with those of these authors, since the use of a dentifrice resulted in a significant increase in lesions at specific locations (buccal and lingual surfaces of the free gingiva). These differences appear to be associated with different degrees of abrasiveness of the toothpaste used. The RDA of an abrasive toothpaste defines its potential on a normalized scale based on accepted standard reference materials. ${ }^{25} \mathrm{RDA}$ is important, as abrasive toothpastes can aggravate brush-induced gingival abrasion. Versteeg et al. ${ }^{24}$ used a dentifrice with an RDA of \pm 77 . In the present study, a dentifrice with a greater abrasiveness (RDA \pm 160 ) was used.

In contrast, the degree of dentifrice abrasion does not influence plaque removal. In 2006 and 2007, Paraskevas et al. ${ }^{26,27}$ found no differences in the reduction of plaque scores using toothpastes with varying RDAs ( \pm 80 to \pm 200 ). Additionally, Rosema et al. ${ }^{22}$ observed that the presence or absence of toothpaste had no effect on plaque removal. The findings of the present study agree with those of these studies, since the presence of a dentifrice did not improve plaque removal.

Studies that evaluated the relationship between soft brushes with end-rounded and tapered bristles on plaque removal and gingival abrasion are scarce. In 2003, Dorfer et al. ${ }^{17}$ observed that brushes with 
tapered bristles removed more plaque compared to those with end-rounded bristles. Using a model of experimental gingivitis, Versteeg et al. ${ }^{8}$ observed that brushes with end-rounded bristles were more effective in reducing plaque and gingival bleeding when compared to those with tapered bristles. These authors also found higher mean values for gingival abrasion with end-rounded bristles, though the differences were not statistically significant. These findings were confirmed in the present study.

The two types of toothbrushes evaluated in the present study were classified as "soft" by their manufacturers. The rounded bristle end had a diameter of $\pm 0.12 \mathrm{~mm}$, whereas the tapered bristle had a diameter of $\pm 0.2 \mathrm{~mm}$ (Table 1, Figure 1). This may indicate that the end-rounded bristles were stiffer and that the tapered bristles underwent greater deflection during brushing, which could explain the difference in our findings regarding plaque removal and gingival abrasion.

Individual variations may influence the evaluation of outcomes, such as plaque removal and gingival abrasion. These variations include brushing methods, force applied during brushing, and gingival tissue characteristics (epithelial thickness). The crossover and split-mouth approaches were used in the present study to reduce these individual variations. Additionally, randomization of contralateral quadrants ( 1 and 3, 2 and 4) ensured equal distribution of strength-related variables with regard to brushing, taking into consideration that all individuals in this study were right-handed, and most likely possessed a greater ability to remove plaque in quadrants 1 and 4. Familiarizing the subjects with the brush types was a methodological measure taken to avoid changes in the brushing method due to the use of brushes of different designs. van der Weijden et al. ${ }^{28}$ observed a reduction of $60 \%$ to $75 \%$ in the incidence of gingival abrasion after a period of familiarization with electric brushes.

Some limitations are present in the current study. First, the sample was comprised of university students, who may have better knowledge of oral health than the overall population. Second, only subjects with a maximum of $15 \%$ of sites with gingival bleeding and with papillas completely filling the interdental spaces were eligible. These characteristics can limit the external validity of the results, requiring caution when extrapolating the results. Third, the severity of gingival abrasion is a more important outcome than the absolute number of lesions. However, the method used for assessing gingival abrasion in the present study is not adequate for this purpose. Histological evaluations should be performed to evaluate the severity of gingival abrasion lesions.

Our results demonstrated that, when used along with a dentifrice, soft toothbrushes with end-rounded bristles removed plaque more effectively compared with brushes with tapered bristles, without resulting in a higher incidence of gingival abrasion. However, the degree of abrasiveness of the toothpaste should also be considered. In the marginal area, the use of an abrasive dentifrice (RDA $= \pm 160$ ) significantly increased the incidence of abrasion, regardless of the toothbrush type used. Although the evidence to confirm the relationship between traumatic toothbrushing and gingival recession are inconclusive, ${ }^{6,29}$ the majority of studies verify this association. ${ }^{1,2,6,29}$ Therefore, individuals who are at risk of developing gingival recession should be cautioned against using a highly abrasive dentifrice.

Long-term studies should be carried out to ascertain whether the presence of gingival abrasion can lead to the development of gingival recession, and also whether these gingival abrasion lesions will remain active over a longer follow-up period.

\section{Conclusion}

Soft toothbrushes with end-rounded bristles removed plaque more effectively than brushes with tapered bristles, without resulting in a higher incidence of gingival abrasion. In the marginal area, the use of a dentifrice $(\mathrm{RDA}= \pm 160)$ significantly increased the incidence of abrasion, regardless of the brush type used. Abrasive dentifrices should be used with caution by individuals who are at risk of developing gingival recession. 


\section{References}

1. Serino G, Wennstrom JL, Lindhe J, Eneroth L. The prevalence and distribution of gingival recession in subjects with a high standard of oral hygiene. J Clin Periodontol. 1994;21(1):57-63. doi:10.1111/j.1600-051X.1994.tb00278.x

2. Daprile G, Gatto MR, Checchi L. The evolution of buccal gingival recessions in a student population: 5-year follow-up. J Periodontol. 2007;78(4):611-4. doi:10.1902/jop.2007.060277

3. Susin C, Haas AN, Oppermann RV, Haugejorden O, Albandar JM. Gingival recession: epidemiology and risk indicators in a representative urban Brazilian population. J Periodontol. 2004:75(10):1377-86. doi:10.1902/jop.2004.75.10.1377

4. Addy M, Hunter ML. Can tooth brushing damage your health? Effects on oral and dental tissues. Inter Dent J. 2003;53(Suppl 3):177-86. doi:10.1111/j.1875-595X.2003.tb00768.x

5. Rosema NAM, Adam R, Grender JM, van der Sluijs E, Supranoto SC, van der Weijden GA. Gingival abrasion and recession in manual and oscillating-rotating power brush users. Int J Dent Hyg. 2014;12(4):257-66. doi: 10.1111/idh.12085

6. Heasman PA, Holliday R, Bryant A, Preshaw PM. Evidence for the occurrence of gingival recession and non-carious lesions as a consequence of traumatic toothbrushing. J Clin Periodontol. 2015;42(Suppl 16):S237-55. doi:10.1111/jcpe.12330

7. Versteeg PA, Rosema NA, Timmerman MF, van der Velden U, van der Weijden GA. Evaluation of two soft manual toothbrushes with different filament designs in relation to gingival abrasion and plaque removing efficacy. Int J Dent Hyg. 2008;6(3):166-73. doi:10.1111/j.1601-5037.2008.00298.x

8. Versteeg PA, Piscaer M, Rosema NA, Timmerman MF, van der Velden U, van der Weijden GA. Tapered toothbrush filaments in relation to gingival abrasion, removal of plaque and treatment of gingivitis. Int J Dent Hyg. 2008;6(3):174-82. doi:10.1111/j.1601-5037.2008.00284.x

9. van der Weijden GA, Hioe KP. A systematic review of the effectiveness of self-performed mechanical plaque removal in adults with gingivitis using a manual toothbrush. J Clin Periodontol. 2005;32(Suppl 6):214-28. doi:10.1111/j.1600-051X.2005.00795.x

10. Carvalho RS, Rossi V, Weidlich P, Oppermann RV. Comparative analysis between hard- and soft-filament toothbrushes related to plaque removal and gingival abrasion. J Clin Dent. 2007;18(3):61-4.

11. Zanatta FB, Bergoli AD, Werle SB, Antoniazzi RP. Biofilm removal and gingival abrasion with medium and soft toothbrushes. Oral Health Prev Dent. 2011;9(2):177-83. doi:10.3290/j.ohpd.a22018

12. Zimmer S, Ozturk M, Barthel CR, Bizhang M, Jordan RA. Cleaning efficacy and soft tissue trauma after use of manual toothbrushes with different bristle stiffness. J Periodontol. 2011;82(2):267-71. doi:10.1902/jop.2010.100328
13. Breitenmoser J, Mörmann W, Mühlemann HR. Damaging effects of toothbrush bristle end form on gingiva. J Periodontol. 1979;50(4):212-6. doi:10.1902/jop.1979.50.4.212

14. Greggianin BF, Oliveira SC, Hass AN, Oppermann RV. The incidence of gingival fissures associated with toothbrushing: crossover 28-day randomized trial. J Clin Periodontol. 2013;40(4):319-26. doi:10.1111/jcpe.12072

15. Niemi ML, Sandholm L, Ainamo J. Frequency of gingival lesions after standardized brushing as related to stiffness of toothbrush and abrasiveness of dentifrice. J Clin Periodontol. 1984;11(4):254-61. doi:10.1111/j.1600-051X.1984.tb02215.x

16. Drisko C, Henderson R, Yancy J. A review of current toothbrush bristle endo-rounding studies. Compend Contin Educ Dent. 1995;16(7):694, 696, 698; quiz 708.

17. Dorfer CE, von Bethlenfalvy ER, Kugel B, Pioch T. Cleaning efficacy of a manual toothbrush with tapered filaments. Oral Health Prev Dent. 2003;1(2):111-8.

18. Tonetti MS, Claffey N; European Workshop in Periodontology group C. Advances in the progression of periodontitis and proposal of definitions of a periodontitis case and disease progression for use in risk factor research. Group C consensus report of the 5th European Workshop in Periodontology. J Clin Periodontol. 2005;32(Suppl 6):210-3. doi:10.1111/j.1600-051X.2005.00822.x

19. Danser MM, Timmerman MF, IJzerman $Y$, Bulthuis $\mathrm{H}$, van der Velden $\mathrm{U}$, van der Weijden GA. Evaluation of the incidence of gingival abrasion as a result of toothbrushing. J Clin Periodontol. 1998;25(9):701-6. doi:10.1111/j.1600-051X.1998.tb02510.x

20. Quigley GA, Hein JW. Comparative cleansing efficiency of manual and power brushing. J Am Dent Assoc. 1962;65(1):26-9. doi:10.14219/jada.archive.1962.0184

21. Turesky S, Gilmore ND, Glickman I. Reduced plaque formation by chloromethyl analogue of vitamin C. J Periodontol. 1970;41(1):41-3. doi:10.1902/jop.1970.41.1.41

22. Rosema NA, Timmerman MF, Versteeg PA, van Palenstein Helderman WH, van der Velden U, van der Weijden GA. Safety and efficacy of two manual toothbrushes. Int J Dent Hyg. 2010;8(4):280-5. doi: 10.1111/j.1601-5037.2010.00475.x

23. Addy M. Measuring success in toothbrush design - an opinion and debate of the concepts. Int Dent J. 1998;48(Suppl 5):509-18. doi:10.1111/j.1875-595X.1998.tb00498.x

24. Versteeg PA, Timmerman MF, Piscaer M, van der Velden U, van der Weijden GA. Brushing with and without dentifrice on gingival abrasion. J Clin Periodontol. 2005;32(2):158-62. doi:10.1111/j.1600-051X.2005.00652.x

25. Hefferren JJ. A laboratory method for assessment of dentrifrice abrasivity. J Dent Res. 1976;55(4):563-73. doi:10.1177/00220345760550040301 
26. Paraskevas S, Timmerman MF, van der Velden U, van der Weijden GA. Additional effect of dentifrices on the instant efficacy of toothbrushing. J Periodontol. 2006;77(9):1522-7. doi:10.1902/jop.2006.050188

27. Paraskevas S, Rosema NA, Versteeg P, Timmerman MF, van der Velden $U$, van der Weijden GA. The additional effect of a dentifrice on the instant efficacy of toothbrushing: a crossover study. J Periodontol. 2007;78(6):1011-6. doi:10.1902/jop.2007.060339

28. van der Weijden GA, Timmerman MF, Piscaer $M$, Snoek I, van der Velden U, Galgut PN. Effectiveness of an electrically active brush in the removal of overnight plaque and treatment of gingivitis. J Clin Periodontol. 2002;29(8):699-704. doi:10.1034/j.1600-051X.2002.290806.x

29. Rajapakse PS, McCracken GI, Gwynnett E, Steen ND, Guentsch A, Heasman PA. Does tooth brushing influence the development and progression of non-inflammatory gingival recession? A systematic review. J Clin Periodontol. 2007;34(12):1046-61. doi:10.1111/j.1600-051X.2007.01149.x 